\title{
MATERNAL LEUKOCYTOSIS AS DIAGNOSTIC MARKERS IN SPONTANEOUSLY DECLARED PRETERM BIRTH
}

\author{
Iliana Koleva-Korkelia ${ }^{1}$
}

\begin{abstract}
Spontaneous preterm birth is the basic problem of perinatal mortality in Bulgaria. Pregnancy is characterized by physiological leukocytosis - determined by twice increased neutrophils levels, unchanged monocyte levels, and a reduction in the levels of lymphocytes, eosinophils, and basophils. The leukocytosis is particularly pronounced in the last trimester, due to physiological stress during pregnancy, and it is a result of the increased inflammatory response, a consequence of selective immune tolerance, immunosuppression, and fetus-immunomodulation.

The objective of this study was to investigate and to appreciate maternal leukocytosis as potential diagnostic markers in spontaneously declared preterm birth and term birth declared cases.

Methods: The case-control study was performed in UMBAL-City Hospital, Stara Zagora during 2017-2020. The study involved 200 patients, split into two groups: G1- the healthy pregnant women, $\mathrm{n}_{1}=100$ with a registered singleton pregnancy, at gestational age $37-39^{+6}$, gave birth on the term (TB); G2- the PTB patients, $\mathrm{n}_{2}=100$, confirmed clinically by cardiotocography for gestation $>32$ weeks. The highly sensitive Leuko-TIC-ELISA-WBC (upper limit of the normal number of Leuc during pregnancy - 15x109 per L) test was used for investigation. Blood samples were taken by puncture of the v.cubitalis in an anticoagulant at the time of hospitalization and examined within $1 \mathrm{hrs}$. Patients with systemic chronic illness and infections were excluded from the study.

The results showed statistically significant Leuc reduction in SPTB patients $(14.31 \pm 2.66 \times 109 / \mathrm{L}, \mathrm{p}>0.03)$, compared to the increased $(14.67 \pm 3.21 \times 109 / \mathrm{L})$ (in normal-15x109L) Leuc values in the TB group.In conclusion, the results of the Leuc value confirm the presence of a systemic leukocytosis/oxidative inflammatory reaction at the time of declaring SPTB and TB.
\end{abstract}

UDC Classification: 618, DOI: https://doi.org/10.12955/pmp.v2.175

Keywords: Leuc, sPTB, TB, leukocytosis

\section{Introduction}

Pregnancy is characterized by physiological leukocytosis (Kaur et al., 2014) - mainly dependent on neutrophils - circulating phagocytes, whose levels double. Kaur et al., (2014) found that other circulating phagocytes (monocytes, lymphocytes, eosinophils, and basophils) retain or suffer a significant decrease in their levels compared to the period before pregnancy. Neutrophil leukocytosis starts from the second month of pregnancy with an ascending trend and is particularly pronounced in the last trimester (Kaur et al.,2014; Akinlaja, 2016). Studies by Kaur et al. (2014) and Akinlaja (2016) commented that leukocytosis is due to physiological stress during pregnancy itself. It is the result of an enhanced inflammatory response due to selective immune tolerance, immunosuppression, and immunomodulation of the fetus. A study by Dhariwal et al. (2016) demonstrated a reduction in lymphocyte levels as the gestational weeks progressed.

In recent years, the study of serological parameters has been used as a major marker for early diagnosis of pregnancy and the assessment of the occurrence of complications such as spontaneous preterm birth (sPTB). The main causes of morbidity and mortality among newborns in Bulgaria are high incidence of sPTB (9.5-10.5\%, National Statistical Institute, 2018). Premature birth is divided into two main types: 1) Idiopathic, spontaneous premature birth (sPTB) (70-80\%) labor is declared with preserved integrity of the amniotic membranes $(50 \%)$ or labor begins after premature rupture of the amniotic sac without declared labor activity $(25-40 \%)$; 2 ) Elective preterm birth (ePTB) $(20-30 \%)$ is declared by induction of labor or by cesarean section as indicated by the mother or fetus before the end of 37 weeks of gestation, (Menon, 2008).

The reasons for the spontaneous declaration of labor and SPTB are not fully understood. The placenta and fetal membranes play a major role in the onset of labor. Intrauterine ascending bacterial infection of the amniotic membranes, waters, placenta, umbilical cord, and the fetus are asymptomatic and paraclinical results are the only confirmation of its presence (Menon, 2019).

Delgado et al. (1994) found that leukocytosis was observed only after a spontaneous normal birth. Balloch and Cauchi (1993) recorded an upper limit of physiological leukocytosis of $-15 \times 10^{9} / \mathrm{L}$. A study by Abbassi-Ghanavati et al. (2009) drew attention to the fact that as pregnancy progresses, the upper limit of the normal Leuc number rises:

\footnotetext{
${ }^{1}$ Trakia University, Medical Faculty, Obstetrics and Gynecology Clinic, Stara Zagora, Bulgaria, iliana_mih@abv.bg
} 
- First trimester $-5.7-13.6 \times 10^{9} / \mathrm{L}$

- Second trimester - $5.6-13.6 \times 10^{9} / \mathrm{L}$

- Third trimester $-5.7-16.9 \times 10^{9} / \mathrm{L}$

\section{Patients and methodology}

The prospective case-control study was conducted in the period 2017-2020, based on cases in the University Hospital by Prof. Dr. St. Kirkovich, Stara Zagora, Bulgaria. It includes 200 women - 100 women aged 20-32 years old giving birth at term (TB) (at 37-39+6 gestation weeks) and 100 women with spontaneous preterm birth (sPTB) at $32-34^{+6}$ gestation weeks. Approval was received from the Ethics Committee (UMHAT "Prof. St. Kirkovich" together with the Faculty of Medicine, 2017/2020 MF, TrU, Stara Zagora, Bulgaria) for the research and all participating mothers/patients gave written informed consent.

Clinical patient information is shown in Table 1. The sample included 200 cases divided into two groups:

- Group $1\left(\mathrm{n}_{2}=100\right)$ women gave birth at term, with active labor at the time of hospitalization.

- Group $2(\mathrm{n} 1=100)$ women with $\mathrm{SPTB}$, admitted with declared active labor and gave birth within 24 hours after hospitalization.

\begin{tabular}{|c|c|c|c|}
\hline Characteristics & $\begin{array}{c}\text { Group } 1(n=100) \\
37-39^{+6} \text { g.week }\end{array}$ & $\begin{array}{c}\text { Group } 2(n=100) \\
32-34^{+6} \text { g.week }\end{array}$ & $* \mathbf{p}$ \\
\hline $\begin{array}{l}\text { Maternal age (y) } \\
\text { Gestational age (w) }\end{array}$ & $\begin{array}{l}26.9 \pm 5.8 \\
38.2 \pm 1.9\end{array}$ & $\begin{array}{l}31.8 \pm 4.12 \\
32.9 \pm 4.12\end{array}$ & $\begin{array}{l}0.043^{\mathrm{a}, \mathrm{LSD} \text { test }} \\
\leq 0.004^{\mathrm{a}, \text { LSD test }}\end{array}$ \\
\hline Body mass index (BMI), kg/m² & $39.6 \pm 2.6$ & $19.3 \pm 0.81$ & $\leq 0.055^{\mathrm{a}, \text { LSD test }}$ \\
\hline $\begin{array}{l}\text { Infections } \\
\text { Tobacco use } \\
\text { Alcohol use }\end{array}$ & $\begin{array}{l}\text { None } \\
\text { None } \\
\text { None }\end{array}$ & $\begin{array}{l}\text { None } \\
\text { None } \\
\text { None }\end{array}$ & $\begin{array}{l}\leq 0.00 \\
\leq 0.00 \\
\leq 0.00\end{array}$ \\
\hline $\begin{array}{l}\text { Abortion } \\
\text { PPROM } \\
\text { Cardio-vascular disease } \\
\text { previous PTB }\end{array}$ & $\begin{array}{l}\text { none } \\
\text { none } \\
\text { NA-not applicable } \\
0.00\end{array}$ & $\begin{array}{l}\text { none } \\
\text { none } \\
\text { NA } \\
0.00(0-2)\end{array}$ & $\begin{array}{l}- \\
- \\
- \\
\leq 0.00\end{array}$ \\
\hline
\end{tabular}

Source: Author

The study included women with singleton pregnancies with live fetuses without ultrasound and biochemical evidence of abnormalities, with preserved integrity of the amniotic membranes at birth and without evidence of diseases complicating the pregnancy. Women with premature rupture of the amniotic sac, preeclampsia, low attachment of the placenta, premature detachment of the placenta, gestational and pre-pregnancy diabetes, anemia, with clinical and paraclinical data on inflammatory and infectious diseases were not included. The gestational age was calculated from the date of the last regular menstruation (LMP) and verified by vaginal speculum examination, and abdominal ultrasonography for measurement (Aloka, Prosound alpha 6) of AFI.

Both groups are characterized by the consumption of additional amounts of folic acid $(\leq 300 \mathrm{mg} / \mathrm{d})$, vitamins ( $\leq 350 \mathrm{mg} / \mathrm{d})$, and magnesium $(\leq 360 \mathrm{mg} / \mathrm{d})$ during pregnancy.

Serological tests of leukocyte levels in the blood were performed during the hospitalization of patients in the laboratory of the University Hospital, Stara Zagora, to increase reliability and minimize the difference in reporting the results. Blood samples from term births were collected in the active phase of birth. After puncture of $\mathrm{v}$. cubitalis within $1 \mathrm{~h}$, blood ( $5 \mathrm{ml} /$ EDTA) samples were centrifuged for 10 minutes at $1600 \mathrm{rpm}$ and plasma was frozen at $80^{\circ} \mathrm{C}$ until analyzed. The reading was performed with the available ELISA assay kits according to the manufacturer's protocols. To interpret the results, an upper limit for the normal number of leukocytes during pregnancy was taken into account $15 \times 10^{9} / \mathrm{L}$. Simultaneously with leukocyte levels, clinical data of each patient were reported: maternal age, gestational week, body fat index, marital status, employment, parity, vaginal bleeding, previous PTB.

\section{Statistical Analysis}

Statistical analysis was performed with Statistic 8.0, Stasoft, Inc., one-way ANOVA, and Student- Ttest to determine the significant difference among data groups. The results were expressed as means \pm standard error (SE). A P value of $\mathrm{p}<0.05$ was considered a statistically significant difference. 


\section{Results and Discussion}

The role of leukocytes as a potential early diagnostic marker for SPTB has been discussed in the current prospective case-control study.

\section{Patient's clinical data}

The clinical data of 100 women who gave birth at term and 100 with sPTB are documented in Table 1. The mean maternal age in group 2 patients with premature uterine contractions ending in PTB was $31.8 \pm 4.12$ years, while the mean age in group 2 women was $26.9 \pm 5.8$ years. Maternal age showed a statistically significant difference $(\mathrm{p} \leq 0.043$, t-test). Consistent with our results, a retrospective study by Newburn-Cook and Onyskiw (2005) emphasizes that older maternal age can be both an independent risk factor and an indicator that contributes to spontaneous PTB. In the opposite study, Auger et al. (2013) commented that the incidence of PTB was sharply increasing among women aged 20-29 years. According to Berghella et al. (2007) gestational age and changes in transvaginal cervical length have a statistically significant effect on the risk of spontaneous PTB. In our study, when comparing the mean gestational age in Group 1 who gave birth at term $(38.2 \pm 1.9$ g.w.) with the mean gestational age in patients in Group 2 with PTB (32.9 \pm 4.12 g.w.), a statistically significant decrease $(\mathrm{p} \leq 0.004, \mathrm{t}$-test $)$ is observed.

The comparison and interpretation of maternal body mass index results against the occurrence of spontaneous PTB or elective PTB should be done with caution due to the scarcity of studies. According to a systematic review by Torloni et al. (2009) it is indicated that women with statistically low pre-existing BMI $(n=520)$ appear to be at increased risk of spontaneous or elective PTB. Additionally, Silva et al. (2019) reported that low BMI early during pregnancy is a major complication associated with medically predicted spontaneous singleton PTB. Consistent with this study, we reported a statistically significant difference in body mass index measurement for both groups, respectively (mean $39.6 \pm 2.6$ in Group 1 vs mean $19.3 \pm 0.81$ in Group 2, $\mathrm{p} \leq 0.055$, t-test). Also, a quantitative statistical relationship between Group 1 and Group 2 was recorded for all three measured factors - maternal age, gestational age, and BMI, respectively (ap <0.003, LSD post hoc test; ap $<0.002$, LSD post hoc test; ap < 0.005, LSD post hoc test). No statistical differences were reported in previous abortion, vaginal bleeding, infectious diseases, tobacco and alcohol use, cardio-vascular disease and previous PTB (NA or $\leq 0.00$, LSD post hoc test).

Liu et al. (2019) reported, in their population-based cohort study, that it is necessary to understand the main mechanisms leading to the risk of PTB, such as a combination of increased or decreased body mass index, obesity or malnutrition before pregnancy, social environment, mother's age, race, place of work, gestational age and ethnicity. Most of the patients included in our study were passive smokers, manual workers living in a low-income social environment, taking folic acid, vitamins, and magnesium irregularly during pregnancy. These findings are consistent with previous observations by D'Silva (2018) and Lamont (2019) where they report that typical predictors of sPTB are insufficient access to prenatal care, low levels of education, low family income, poor nutritional status, increased maternal age, and systemic active or passive smoking.

\section{Comparison of the Leuco levels in TB and SPTB patients}

Strong emotional and oxidative stress, pathological infections in the body, regular starvation, and pregnancy lead to an increased number of white blood cells $\left(15 \times 10^{9} / \mathrm{L}\right)$ and the registration of leukocytosis. In previous studies by Silva et al. (2019), D'Silva (2018), and Lamont (2019), pregnancyhas been shown to cause a variety of physiological changes in the body associated with the suppression of humoral and cellular immune functions. Bartkeviciene et al., (2013) in their investigation noted that the CD8 T lymphocytes percentages significantly increased and monocytes decreased during the third trimester. In the present study, examining pregnant women from Southern Bulgaria, we discussed and compared the serological level of leukocytes as a potential reliable diagnostic marker for SPTB (Figure 1).

After the statistical processing of the results, we found that the levels of leukocytes in the mother's blood in spontaneous premature birth, and at term delivery are within the normal physiological leukocytosis characteristic of the third trimester of pregnancy. In term births, leukocytosis was slightly higher at birth relative to the maximum reference value (mean $14.67 \pm 3.21 \times 10^{9} / \mathrm{L}$ compared to mean $\left.15 \times 10^{9} / \mathrm{L}\right)$. 


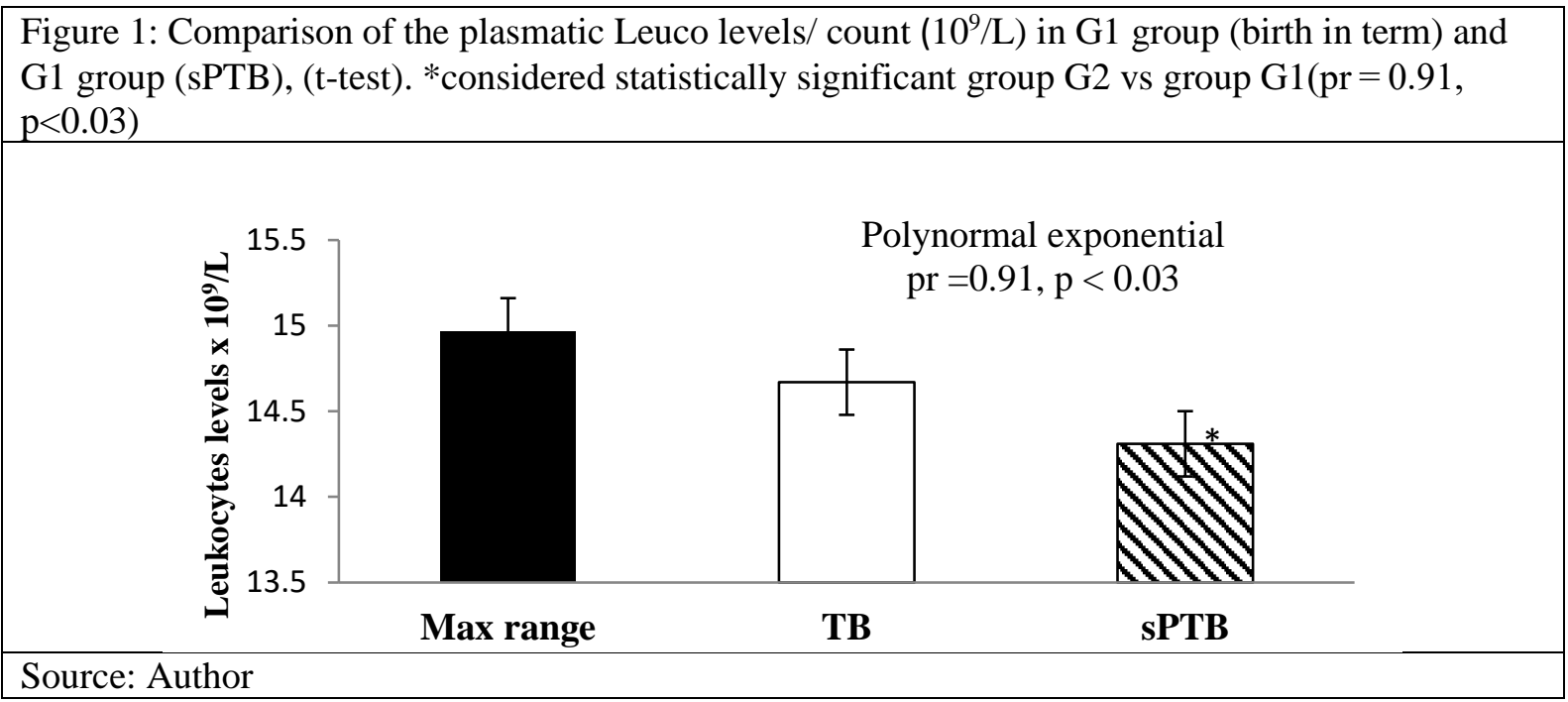

Lower leukocyte values than the reference values were registered in women with spontaneous premature birth (mean $14.31 \pm 2.66 \times 109 / \mathrm{L}$ ). Moreover, in $85 \%$ of patients with PTB, there was a statistically significant decrease in the level of leukocytes $(\mathrm{r}=0.953, \mathrm{p}<0.03$, LSD post hoc test). Besides, a positive correlation $(\mathrm{p}=0.05)$ between the number of leukocytes in maternal blood in $\mathrm{TB}$ and PTB was identified. There is a significant correlation between elevated Leuco levels in TB and sPTB. Circulating leukocytosis is almost commensurate with the maximum prognostic value $=$ $15 \times 10^{9} / \mathrm{L}$ for this indicator in the absence of clinical signs of infectious inflammation in the body (Zhang et al., 2017).

Zhang et al. (2017) by our findings confirm that leukocytes in the cervix, myometrium, and decidua reach a maximum level immediately before birth, even in the absence of infection. According to Qiu et al. (2019), an increase in leukocyte/WBC levels is a likely indicator of the presence of physiological, biochemical, and oxidative abnormalities in the mother, leading to inappropriate activation of inflammatory pathways and a metabolic syndrome that is probably impossible to control at PTB. Therefore, the premature accumulation of leukocytes abruptly infiltrates the uterine tissues and promotes maturation and contractile activity of the uterus, leading to spontaneous premature birth and expulsion of the baby (Zhang et al., 2017)

\section{Conclusion}

PTB forecasting remains a contentious issue. We found that increased leukocytosis, both in women who gave birth at term and for patients with spontaneous PTB symptoms. The early spontaneous PTB symptoms are mainly based on gestational age. Further studies will be needed to focus on oxidative stress inflammation in the presence of leukocytosis and to determine the pathways that leukocytosis can surf as an early diagnosis biomarker of spontaneous PTB.

Acknowledgements. The study was supported by the PhD program of Dr. Iliana Koleva-Korkelia and by scientific project 1/2020 of Medical Faculty, Trakia University, Bulgaria.

\section{References}

Abbassi-Ghanavati, M., Greer, L. G., \& Cunningham, F. G. (2009). Pregnancy and laboratory studies: a reference table for clinicians. Obstetrics \& Gynecology, 114(6), 1326-1331.

Akinlaja, O. (2016). Hematological changes in pregnancy-The preparation for intrapartum blood loss. Obstet Gynecol Int $J, 4(3), 00109$.

Auger, N., Hansen, A. V., \& Mortensen, L. (2013). Contribution of maternal age to preterm birth rates in Denmark and Quebec, 1981-2008. American journal of public health, 103(10), e33-e38.

Balloch, A. J., \& Cauchi, M. N. (1993). Reference ranges for haematology parameters in pregnancy derived from patient populations. Clinical \& Laboratory Haematology, 15(1), 7-14.

Bartkeviciene, D., Pilypiene, I., Drasutiene, G., Bausyte, R., Mauricas, M., Silkunas, M., \& Dumalakiene, I. (2013). Leukocytosis as a prognostic marker in the development of fetal inflammatory response syndrome. Libyan Journal of Medicine, 8(1)

Berghella, V., Roman, A., Daskalakis, C., Ness, A., \& Baxter, J. K. (2007). Gestational age at cervical length measurement and incidence of preterm birth. Obstetrics \& Gynecology, 110(2 Part 1), 311-317. 
Delgado, I., Neubert, R., \& Dudenhausen, J. W. (1994). Changes in white blood cells during parturition in mothers and newborn. Gynecologic and obstetric investigation, 38(4), 227-235.

Dhariwal, S. K., Narang, S., Singh, A., \& Nema, S. (2016). Evaluation of haematological indices, neutrophils and platelets in pregnant women attending tertiary care centre. Indian Journal of Pathology and Oncology, 3(2), 297-304.

D'Silva, A. M. (2018). Identification of first trimester maternal serum markers predictive of spontaneous preterm birth. Western Sydney University, Australia.

Esplin, M. S. (2014). Overview of spontaneous preterm birth: a complex and multifactorial phenotype. Clinical obstetrics and gynecology, 57(3), 518-530.

Kaur, S., Khan, S., \& Nigam, A. (2014). Hematological profile and pregnancy: a review. Int J Adv Med, 1(2), 68-70.

Lamont, R. F. (2019). Spontaneous preterm labour that leads to preterm birth: An update and personal reflection. Placenta, 79, 21-29.

Liu, B., Xu, G., Sun, Y., Du, Y., Gao, R., Snetselaar, L. G., ... \& Bao, W. (2019). Association between maternal prepregnancy obesity and preterm birth according to maternal age and race or ethnicity: a population-based study. The Lancet Diabetes \& Endocrinology, 7(9), 707-714.

Menon, R. (2008). Spontaneous preterm birth, a clinical dilemma: etiologic, pathophysiologic and genetic heterogeneities and racial disparity. Acta obstetricia et gynecologica Scandinavica, 87(6), 590-600.

Menon, R. (2019). Initiation of human parturition: signaling from senescent fetal tissues via extracellular vesicle mediated paracrine mechanism. Obstetrics \& gynecology science, 62(4), 199.

Newburn-Cook, C. V., \& Onyskiw, J. E. (2005). Is Older Maternal Age a Risk Factor for Preterm Birth and Fetal Growth Restriction? A SystematicReview. Health care for women international, 26(9), 852-875.

Qiu, L., Pan, M., Zhang, R., \& Ren, K. (2019). Maternal peripheral blood platelet-to-white blood cell ratio and platelet count as potential diagnostic markers of histological chorioamnionitis-related spontaneous preterm birth. Journal of clinical laboratory analysis, 33(4), e22840.

Silva, F. P., Souza, R. T., Cecatti, J. G., Passini, R., Tedesco, R. P., Lajos, G. J., ... \& Silva, C. M. (2019). Role of Body Mass Index and gestational weight gain on preterm birth and adverse perinatal outcomes. Scientific reports, 9(1), 1-12.

Torloni, M. R., Betran, A. P., Daher, S., Widmer, M., Dolan, S. M., Menon, R., ... \& Merialdi, M. (2009). Maternal BMI and preterm birth: a systematic review of the literature with meta-analysis. The Journal of Maternal-Fetal \& Neonatal Medicine, 22(11), 957-970.

Zhang, J., Shynlova, O., Sabra, S., Bang, A., Briollais, L., \& Lye, S. J. (2017). Immunophenotyping and activation status of maternal peripheral blood leukocytes during pregnancy and labour, both term and preterm. Journal of cellular and molecular medicine, 21(10), 2386-2402. 